\title{
Plasma Amino Acid Differences in Very Low Birth Weight Infants Fed either Human Milk or Whey-Dominant Cow Milk Formula
}

\author{
RICHARD J. SCHANLER AND CUTBERTO GARZA \\ Sections of Neonatology and of Nutrition and Gastroenterology and USDA/ARS Children's Nutrition Research \\ Center, Department of Pediatrics, Baylor College of Medicine and Texas Children's Hospital,
} Houston, Texas 77030

\begin{abstract}
Midmorning plasma amino acid levels were measured in 31 healthy, very low birth weight infants (mean age 16 days, mean birth weight $1180 \mathrm{~g}$, gestation 29 wk) during 96-h balance studies. All infants received continuous enteral infusion of isonitrogenous, isocaloric preparations of either human milk fortified with pasteurized, lyophilized fractions of mature human milk $(n=18)$ or whey-dominant cow milk-based formula $(n=13)$. Weight gain $(15 \mathrm{~g} / \mathrm{kg} /$ day $)$, nitrogen retention $(303 \mathrm{mg} / \mathrm{kg} /$ day $)$, and metabolizable energy $(104 \mathrm{kcal} / \mathrm{kg} /$ day $)$ were similar between groups. Plasma levels of threonine, valine, and the sum of essential amino acids were significantly greater in the whey-dominant formula-fed infants $(p<0.01)$. Taurine and cystine were measured in significantly greater concentrations in the fortified human milk and threonine, valine, methionine, and lysine in the whey-dominant cow milk formula $(p<0.01)$. Relationships between plasma amino acid levels and indices of nitrogen utilization differed between groups. These differences suggest that further modifications of whey-dominant formulas may be indicated. (Pediatr Res 21: 301-305, 1987)
\end{abstract}

\section{Abbreviations}

VLBW, very low birth weight

BUN, blood urea nitrogen

When VLBW infants are fed a cow milk-based formula, a whey- rather than a casein-dominant formulation is recommended often $(1,2)$. The assumption implicit in this recommendation is that an increased proportion of bovine milk whey in the formula's protein substantially increases the similarity between the formula and human milk (2). Differences in the proteins found in human and bovine wheys, however, weaken this premise. The predominant proteins in human whey are $\alpha$ lactalbumin, $\operatorname{SIgA}$, and lactoferrin; those predominant in bovine whey are $\beta$-lactoglobulin, IgG, and $\alpha$-lactalbumin. The amino

Received July 23, 1986; accepted October 29, 1986.

This work is a publication of the USDA/ARS Children's Nutrition Research Center, Department of Pediatrics, Baylor College of Medicine and Texas Children's Hospital. This project has been funded in part with federal funds from the U.S. Department of Agriculture, Agricultural Research Service under Cooperative Agreement $58-7 \mathrm{MNI}-6-100$. The contents of this publication do not necessarily reflect the views or policies of the U.S. Department of Agriculture, nor does mention of trade names, commercial products, or organizations imply endorsement by the U.S. Government.

Address all correspondence and reprint requests to Richard J. Schanler, M.D. Children's Nutrition Research Center, 6608 Fannin, Suite 601, Houston, TX 77030 acid compositions of human and bovine whey proteins differ ( 3 , 4), as do those of the casein fractions in human and bovine milk (3). Furthermore, the biological functions associated with human whey proteins, e.g. SIgA and lactoferrin, may affect protein utilization and complicate the interpretation of compositional data.

Plasma amino acid levels have been studied as indicators of protein quality and general protein homeostasis in infants fed a variety of milks (5-7). Highly variable levels of amino acids have been reported in these studies and may reflect differences in feeding protocols, timing of plasma sampling relative to feedings, clinical conditions of infants, interindividual variability in human milk composition, and the possible effects of distinct formula processing protocols on the rates of digestion of milk proteins. Nonetheless, various investigators have reported that values for serum total proteins, serum albumin, plasma amino acids, BUN, and urine osmolality of infants fed whey-dominant formulas resemble those of infants fed human milk more closely than those of infants fed casein-dominant formulas $(5,8-11)$.

Nitrogen from whey-dominant formulas appears to be used more efficiently for growth than that from casein-dominant formulas $(2,6)$. An evaluation of the similarity between responses to human milk and casein- or whey-dominant formulas is complicated, however, because feeding comparisons have seldom been performed with isonitrogenous and isocaloric diets. This characteristic of previous investigations affects assessments of protein quality most directly.

Our study was designed to measure amino acid intakes and plasma levels in VLBW infants fed isonitrogenous, isocaloric diets of human milk or bovine whey-dominant formula and to assess the relationship between these values and growth, nitrogen utilization, and selected biochemical markers of nutritional status in this population.

\section{METHODS}

Study design. Thirty-one VLBW infants were enrolled in a longitudinal study after informed consent was obtained from their parents. The protocol and consent forms were approved by the Institutional Review Boards for Human Research of Baylor College of Medicine and Texas Children's Hospital. Infants were enrolled during the 1st wk of life, assigned to a human milk or formula group based on parental choice, and enteral feeding was instituted. The infants were fed either their mothers' milk fortified with lyophilized fractions of skim and cream derived from pasteurized $\left(72^{\circ} \mathrm{C}, 15 \mathrm{~s}\right)$, mature human milk or a whey-dominant cow-milk formula. The formula group received Similac PM 60/40 (Ross Laboratories, Columbus, OH). Infants maintained their respective feeding regimens from the time enteral feeding was initiated to the study's conclusion. Growth, metabolic re- 
sponses, and nutrient balances were measured (12). A 96-h nutrient balance study was performed at 2 to $3 \mathrm{wk}$ of life to estimate metabolizable energy intake and the absorption and retention of nitrogen and minerals. Plasma amino acids, total protein, albumin, prealbumin, BUN, and whole blood hemoglobin were measured during the balance study.

To ensure that the human milk and formula were isonitrogenous and isocaloric, the human milk was fortified with quantities of human milk-derived fractions of skim and cream based on the energy and nitrogen contents of the formula. These components were added to an aliquot of fresh milk sufficient for 1 day's feedings and were determined in our laboratory from weekly analyses of each mother's milk and from analyses of each lot of formula. Details of the preparation and processing of human milk have been reported (12). Infants received a controlled intake of $125 \mathrm{ml} / \mathrm{kg} /$ day $(125 \mathrm{kcal} / \mathrm{kg} /$ day $)$ administered by continuous nasogastric infusion from the onset of enteral feeding to the end of the balance study. A syringe infusion pump (Auto Syringe, Hookset, NH) was used to minimize losses from the nonhomogeneity of the milk. Multivitamins (Poly-Vi-Sol, Mead Johnson Co., Evansville, IN) and $400 \mathrm{IU}$ vitamin D (Drisdol, Winthrop Laboratories, New York, NY), and $100 \mathrm{mg} / \mathrm{kg} / \mathrm{day}$ of an oral preparation of vitamin $\mathrm{E}$ were administered daily.

Patient selection. Healthy, VLBW infants between 28 and 30 wk gestation were selected from the neonatal nurseries of Texas Children's Hospital based on the following criteria: appropriate growth for gestational age; absence of major congenital anomalies or sustained cardiopulmonary, infectious, and gastrointestinal diseases; and achievement of full enteral feeding by 15 days of life. The two groups of infants were similar with respect to birth weight, gestational age, study age, nitrogen intake and retention, metabolizable energy intake, and selected biochemical markers of nutritional status (Table 1). Weight gain and increments in length and head circumferences were similar between groups. These data have been reported previously (12). Enteral nutrition was instituted at $6 \pm 2$ and $5 \pm 2$ days of life and complete enteral nutrition was achieved at $14 \pm 3$ and $13 \pm 2$ days in the fortified human milk and formula groups, respectively. In the 1 st wk of life, serum total protein, $4.0 \pm 0.4$ versus $4.2 \pm 0.5$, and whole blood hemoglobin values, $14.2 \pm 2.7$ versus $14.5 \pm$ 2.7 , were similar in fortified human milk and formula groups, respectively.

Chemical methods. Heparinized arterial or arterialized capillary blood samples for the determination of plasma proteins and amino acids were obtained between 0800 and $1200 \mathrm{~h}$ on the $3 \mathrm{rd}$ or 4th day of the balance study. Plasma was separated immediately and samples were stored for analysis at $-70^{\circ} \mathrm{C}$. Our treatment of blood samples does not provide reliable estimates of plasma cystine, therefore, these values are not reported (13). The methods for plasma protein determinations have been re-

Table 1. Characteristics of study infants (mean $\pm S D$ )

\begin{tabular}{lcc}
\hline \multicolumn{1}{c}{ Characteristic } & $\begin{array}{c}\text { Fortified } \\
\text { human milk } \\
(n=18)\end{array}$ & $\begin{array}{c}\text { Whey-dominant } \\
\text { cow-milk } \\
\text { formula } \\
(n=13)\end{array}$ \\
\hline Birth wt (g) & $1176 \pm 146$ & $1184 \pm 110$ \\
Gestational age (wk) & $29 \pm 0.7$ & $29 \pm 0.6$ \\
Study age (day) & $17 \pm 3$ & $16 \pm 2$ \\
Wt gain (g/kg/day) & $16 \pm 5$ & $14 \pm 5$ \\
Nitrogen intake (mg/kg/day) & $459 \pm 42$ & $445 \pm 34$ \\
Nitrogen retention (mg/kg/day) & $302 \pm 48$ & $304 \pm 23$ \\
Metabolizable energy (kcal/kg/day) & $106 \pm 12$ & $102 \pm 7$ \\
Biochemical values & & \\
$\quad$ Total protein (g/dl) & $4.9 \pm 0.3$ & $4.7 \pm 0.3$ \\
Albumin $(\mathrm{g} / \mathrm{dl})$ & $3.4 \pm 0.4$ & $3.3 \pm 0.4$ \\
Prealbumin $(\mathrm{mg} / \mathrm{dl})$ & $12.4 \pm 3.6$ & $12.0 \pm 2.8$ \\
Hemoglobin $(\mathrm{g} / \mathrm{dl})$ & $14.3 \pm 2.9$ & $12.8 \pm 2.3$ \\
\hline
\end{tabular}

ported (12). For measurement of amino acids, plasma was treated with $0.5 \%(\mathrm{w} / \mathrm{v})$ sodium dodecylsulfate and deproteinized with sulfosalicylic acid in lithium citrate buffer adjusted to $\mathrm{pH} 2.8$ (14). The Beckman 121MB Amino Acid Analyzer (Beckman Instruments Inc., Palo Alto, CA) was used for these measurements.

Aliquots from the day's milk supply were removed and stored for analysis at $-70^{\circ} \mathrm{C}$. Samples were defatted, hydrolyzed with $6 \mathrm{~N} \mathrm{HCl}$ for $24 \mathrm{~h}$ under nitrogen, neutralized, and filtered before analysis (15). This method does not measure milk ornithine asparagine, citrulline, hydroxyproline, or tryptophan. Because of degradation, glutamine and glutamic acid are reported as a combined value. Taurine in milk was determined on defatted samples after precipitation of proteins. The methods for detection of total nitrogen and energy have been described (12).

Data analysis. Plasma and milk amino acid concentrations were distributed normally. Comparisons between feeding groups were performed by Student's $t$ test. Relationships were tested by multiple linear regression. The level of significance was set at $p$ $<0.01$ because multiple testing was performed.

\section{RESULTS}

Plasma concentrations of threonine, valine, and the sum of the essential amino acids were significantly greater in the infants fed the whey-dominant formula (Table 2). No differences were detected between groups in the sum of the total plasma amino acids, the plasma ratio of glycine/valine; the ratio of tryptophan/ sum of tyrosine, phenylalanine, leucine, isoleucine, and valine; and the sums of branched-chain, aromatic, and sulfur-containing amino acids.

Whereas concentrations of taurine and cystine were significantly greater in the fortified human milk (Table 3 ), those of threonine, methionine, lysine, and valine were significantly

Table 2. Plasma amino acid $(A A)$ concentrations in premature infants fed fortified human milk or whey-dominant cow-milk formula (mean \pm SEM)

\begin{tabular}{lccc}
\hline \multicolumn{1}{c}{$\begin{array}{c}\text { AA } \\
\text { ( } \mu \text { mol/liter })\end{array}$} & $\begin{array}{c}\text { Fortified } \\
\text { human milk } \\
(n=18)\end{array}$ & $\begin{array}{c}\text { Whey-dominant } \\
\text { cow-milk } \\
\text { formula } \\
(n=13)\end{array}$ & $p$ \\
\hline Taurine & $82 \pm 10$ & $73 \pm 15$ & 0.630 \\
Threonine* & $98 \pm 7$ & $389 \pm 38$ & 0.000 \\
Serine & $130 \pm 11$ & $198 \pm 28$ & 0.039 \\
Proline & $132 \pm 11$ & $164 \pm 16$ & 0.110 \\
Glycine & $188 \pm 21$ & $263 \pm 35$ & 0.087 \\
Alanine & $180 \pm 18$ & $253 \pm 35$ & 0.079 \\
Valine* & $85 \pm 6$ & $124 \pm 12$ & 0.011 \\
Methionine* & $26 \pm 4$ & $44 \pm 9$ & 0.086 \\
Isoleucine* & $43 \pm 5$ & $55 \pm 6$ & 0.170 \\
Leucine* & $83 \pm 7$ & $88 \pm 8$ & 0.660 \\
Tyrosine* & $67 \pm 7$ & $66 \pm 9$ & 0.960 \\
Phenylalanine* & $38 \pm 3$ & $48 \pm 6$ & 0.210 \\
Lysine* & $95 \pm 8$ & $119 \pm 14$ & 0.170 \\
Histidine* & $57 \pm 4$ & $55 \pm 7$ & 0.820 \\
Arginine & $58 \pm 7$ & $47 \pm 10$ & 0.370 \\
Aspartic acid & $11 \pm 2$ & $21 \pm 5$ & 0.130 \\
Ornithine & $77 \pm 9$ & $86 \pm 12$ & 0.520 \\
Tryptophan* & $24 \pm 4$ & $34 \pm 5$ & 0.130 \\
Asparagine & $38 \pm 7$ & $71 \pm 12$ & 0.030 \\
Hydroxyproline & $43 \pm 3$ & $49 \pm 5$ & 0.240 \\
Glutamic acid-glutamine & $422 \pm 43$ & $367 \pm 61$ & 0.480 \\
Essential AA & $654 \pm 157$ & $1028 \pm 33$ & 0.003 \\
Total AA & $2040 \pm 599$ & $2602 \pm 1067$ & 0.120 \\
E/T ratio & 0.33 & 0.41 & 0.001 \\
\hline
\end{tabular}

* Essential AA. 
greater in the whey-dominant formula (Table 3). The formula also had greater concentrations of serine, glutamic acid plus glutamine, and isoleucine but these differences were not statistically significant, $0.05>p>0.01$. No significant differences were detected between milks in the concentrations of total essential, branched-chain, aromatic, or sulfur-containing amino acids.

Associations between amino acid intake and plasma amino acid levels were not detected within feeding groups. When groups were combined, however, plasma levels were associated positively with the intake of the respective amino acid for threonine, serine, alanine, and the sum of aromatic and essential amino acids ( $r=$ 0.49 to $0.64, p<0.01$ ). In addition, relationships of borderline statistical significance $(0.05>p>0.01)$ were observed for proline, valine, leucine, tyrosine, phenylalanine, lysine, histidine, arginine, and asparagine.

Distinct associations were noted within each feeding group between plasma amino acid levels and indices of nitrogen utilization and other markers of nitrogen metabolism. Positive correlations were observed in the fortified human milk group between plasma lysine and serum total protein (Fig. 1, $r=0.66, p$ $<0.01$ ). By linear regression analysis, the plasma lysine level accounted for $44 \%$ of the variability in serum total protein levels. The following positive correlations were observed in the formula group ( $r=0.71$ to $0.74, p<0.01)$ : nitrogen retention and plasma glycine, tryptophan, and alanine (Fig. 2); nitrogen utilization (\% $\mathrm{N}$ retention/intake) and plasma isoleucine and phenylalanine; and $\mathrm{N}$ absorption and plasma arginine. Plasma glycine, tryptophan, and alanine, together, accounted for $82 \%$ of the variability in nitrogen retention in the formula group. Negative correlations $(r=-0.71$ to $-0.81, p<0.01)$ were observed between whole blood hemoglobin level and the plasma levels of isoleucine, glycine, alanine, proline, leucine (Fig. 3), and total and essential amino acids. The five amino acids accounted for $89 \%$ of the variability in whole blood hemoglobin level in the formula group.

\section{DISCUSSION}

VLBW infants received isonitrogenous, isocaloric preparations of either fortified human milk or whey-dominant cow-milk

Table 3. Concentrations of amino acids (AA) in fortified human milk and whey-dominant cow-milk formula (mean \pm SEM)

\begin{tabular}{|c|c|c|c|}
\hline $\begin{array}{c}\mathrm{AA} \\
(\mu \mathrm{mol} / \text { liter })\end{array}$ & $\begin{array}{c}\text { Fortified } \\
\text { human milk } \\
(n=18)\end{array}$ & $\begin{array}{l}\text { Whey-dominant } \\
\text { cow-milk } \\
\text { formula } \\
(n=11)\end{array}$ & $p$ \\
\hline Taurine & $384 \pm 31$ & $205 \pm 37$ & 0.001 \\
\hline Threonine* & $5725 \pm 324$ & $8370 \pm 532$ & 0.001 \\
\hline Serine & $6283 \pm 492$ & $8627 \pm 729$ & 0.016 \\
\hline Proline & $10097 \pm 610$ & $12174 \pm 985$ & 0.091 \\
\hline Glycine & $4883 \pm 309$ & $4442 \pm 372$ & 0.370 \\
\hline Alanine & $6307 \pm 365$ & $7395 \pm 674$ & 0.180 \\
\hline Valine* & $6410 \pm 487$ & $8445 \pm 586$ & 0.014 \\
\hline Cystine* & $578 \pm 56$ & $250 \pm 72$ & 0.002 \\
\hline Methionine* & $1512 \pm 85$ & $2600 \pm 224$ & 0.000 \\
\hline Isoleucine* & $5350 \pm 300$ & $6836 \pm 499$ & 0.021 \\
\hline Leucine* & $10886 \pm 727$ & $12304 \pm 1130$ & 0.310 \\
\hline Tyrosine* & $3783 \pm 222$ & $3324 \pm 341$ & 0.270 \\
\hline Phenylalanine* & $3479 \pm 205$ & $3858 \pm 309$ & 0.320 \\
\hline Lysine* & $6598 \pm 435$ & $9642 \pm 871$ & 0.007 \\
\hline Histidine* & $2130 \pm 143$ & $2387 \pm 202$ & 0.310 \\
\hline Arginine & $2963 \pm 206$ & $2609 \pm 254$ & 0.290 \\
\hline Aspartic acid & $9584 \pm 661$ & $11517 \pm 938$ & 0.110 \\
\hline Glutamic acid-glutamine & $17241 \pm 815$ & $22798 \pm 1872$ & 0.017 \\
\hline Essential AA & $47226 \pm 10276$ & $58016 \pm 14610$ & 0.049 \\
\hline Total AA & $107700 \pm 22106$ & $127784 \pm 33196$ & 0.100 \\
\hline $\mathrm{E} / \mathrm{T}$ ratio & 0.45 & 0.45 & \\
\hline
\end{tabular}

* Essential AA.

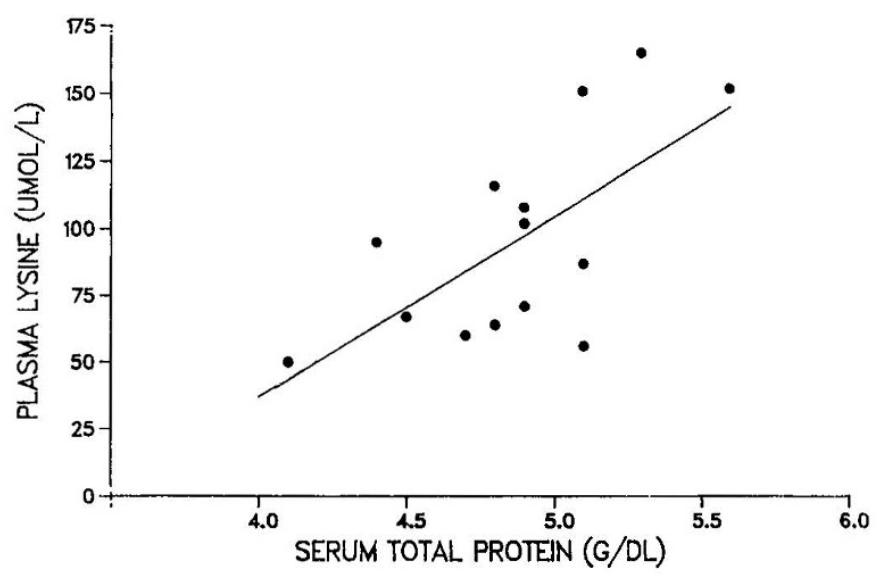

Fig. 1. The relationship between plasma lysine and serum total protein in fortified human milk-fed infants. $\mathrm{Y}=-231+67.1 \mathrm{X}, r=0.66, p$ $<0.01$, where $\mathrm{Y}$ in $\mu \mathrm{mol} / \mathrm{liter}=$ plasma lysine and $\mathrm{X}$ in $\mathrm{g} / \mathrm{dl}=$ serum total protein.

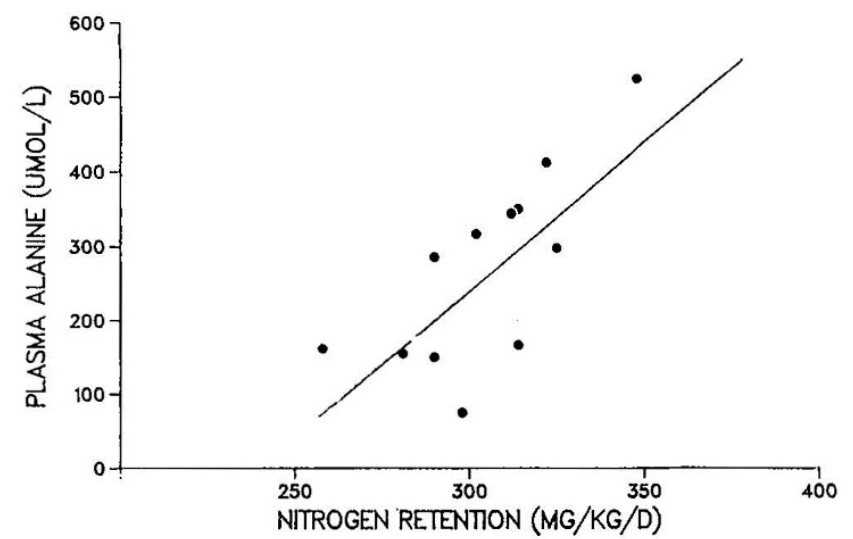

Fig. 2. The relationship between plasma alanine and nitrogen retention in whey-dominant cow milk formula-fed infants. This relationship is representative of those observed in this group between several plasma amino acids and indices of nitrogen metabolism; see text for details. Y $=-941+3.9 \mathrm{X}, r=0.71, p<0.01$, where $\mathrm{Y}$ in $\mu \mathrm{mol} /$ liter $=$ plasma lysine and $X$ in $\mathrm{mg} / \mathrm{kg} /$ day $=$ nitrogen retention.

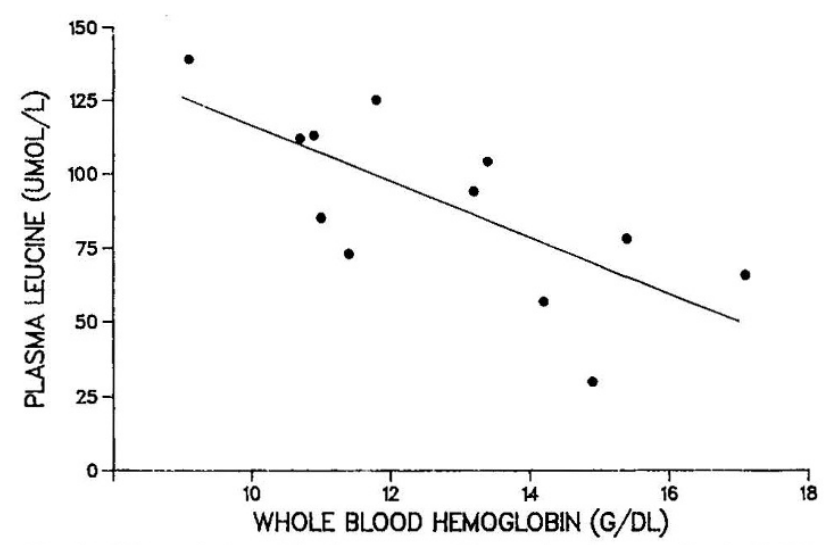

Fig. 3. The relationship between plasma leucine and whole blood hemoglobin in whey-dominant cow milk formula-fed infants. This negative relationship is representative of those observed in this group between several plasma amino acids and whole blood hemoglobin; see text for details. $\mathrm{Y}=211-9.5 \mathrm{X}, r=0.72, p<0.01$, where $\mathrm{Y}$ in $\mu \mathrm{mol} / \mathrm{liter}$ $=$ plasma leucine and $\mathrm{X}$ in $\mathrm{g} / \mathrm{dl}=$ whole blood hemoglobin. 
formula and demonstrated similar growth rates and gross indices of nitrogen utilization (12). Growth and nitrogen retentions were similar to those observed in utero at corresponding gestational stages. Plasma amino acid levels differed, however, between feeding groups. These differences were largely a reflection of amino acid intakes. Variations in the amino acid compositions of the nutrient sources were responsible for these differences. Our fortified human milk preparation, when compared with published values for pooled human milk, resulted in approximately 20 to $30 \%$ increases in the content of most amino acids $(5,16)$. This increment parallels the augmentation in total nitrogen concentration measured after fortification of human milk (12).

Appropriate protein intakes based on plasma amino acid levels are difficult to establish for VLBW infants because standards have not yet been determined. This is particularly true for infants fed by continuous enteral infusion. Plasma levels may be compared with those reported for umbilical cord blood obtained during the delivery of full-term infants (17) or with values reported for fetal cord blood obtained between 19 and 29 wk of gestation (18). In our study, plasma levels of threonine, alanine, lysine, and valine of infants fed fortified human milk and plasma lysine of infants fed the whey-dominant formula were below the minimum values measured in the umbilical artery at term. Plasma hydroxyproline levels in both groups and serine levels in the formula-fed infants were greater than the maximum values measured in the umbilical artery at term. Whether the high hydroxyproline levels reflected an increased turnover of bone in the groups studied is not clear.

Infants fed fortified human milk had threonine and alanine values below $1 \mathrm{SD}$ of the mean values reported for measurements of the umbilical artery at 19 to 29 wk gestation; both fortified human milk- and formula-fed infants had taurine, lysine, histidine, and valine values that were 1 SD below these mean values. None of the values obtained was significantly above those reported for the equivalent stage of intrauterine development. Whether these lower levels reflected amino acid deficiency remains a question. Of interest from a functional standpoint, however, is our finding that VLBW infants appear unable to maintain the levels of serum proteins measured at birth (12). Infants in our study demonstrated declines in serum proteins which usually are ascribed to hepatic immaturity (12). An alternate explanation suggested by these comparisons is that the quantities of dietary amino acids may be significantly less than those supplied in utero and/or that the pattern of amino acids supplied by both fortified human milk or whey-dominant formula is significantly different from the optimal pattern required to maintain serum proteins.

An alternative manner to assess protein intake is to compare the plasma levels of these infants with those of infants fed pooled human milk in later periods of postnatal life. Such comparisons are complicated by differences in intakes, sampling times of plasma, and postnatal ages. Possibly the closest comparison may be made to values reported for infants fed pooled human milk every 1 to $2 \mathrm{~h}$ and in whom plasma values were obtained randomly $(5,9,10,16,19)$. Again, threonine and valine levels in plasma of infants who were fed fortified human milk fell slightly below $1 \mathrm{SD}$ of the mean value measured in the comparison group.

Differences in plasma amino acid levels may reflect possible dissimilarities in the chemical maturity of the two feeding groups; this is unlikely. The parallel characteristics of the two groups outlined in Table 1, similarities in the times enteral feeding was initiated and full enteral feedings were maintained, and comparable initial concentration values for serum total proteins and whole blood hemoglobin are evidence for the groups' homogeneity. Disparities in plasma levels of amino acids most likely reflect differences in diet. This view is supported by relationships between amino acid intakes and plasma levels of amino acids.

The functional significance of plasma amino acid levels may be assessed by examining relationships between these measurements and other indices of nitrogen metabolism. We found that the group fed fortified human milk had lysine levels which correlated positively with total serum proteins, suggesting that lysine levels were not adequate for the needs of this group. In contrast, we found many more associations between plasma amino acid levels and indices of nitrogen metabolism in the whey-dominant formula-fed group. The positive associations between selected amino acids and nitrogen retention suggested that increases in the intakes of these amino acids may have resulted in greater retentions of nitrogen, whereas the negative associations between hemoglobin and selected amino acids suggested that the pattern of amino acids was either inadequate or that the amount provided was excessive. There may be more support for the former assumption because low hemoglobin levels in VLBW infants have been related to inadequate intakes of protein $(20)$. We might speculate that plasma levels would be stable relative to other functions when the intake is neither excessive nor deficient (21). Therefore, were amino acid or protein intakes optimal, positive, or negative relationships would be unlikely between the plasma amino acid levels and other measures of nitrogen metabolism.

Growth velocity and nitrogen balance have been identified as important determinants of plasma amino acid levels (6). If these criteria are used as the principal determinants of adequate protein sources, then either fortified human milk or whey-dominant formula qualifies, and intrauterine rates of growth and nitrogen retention can be achieved (12).

However, the groups fed fortified human milk and wheydominant formula differed in their plasma concentrations of specific amino acids and in the relationships between these levels and other indices of nitrogen metabolism. The negative associations detected between plasma amino acid levels and hemoglobin values and the positive associations between selected plasma amino acid levels and nitrogen retention leads to the speculation that the amino acid pattern of the whey-dominant formula may be unbalanced. The relationships between plasma amino acid levels and serum total protein and whole blood hemoglobin in infants fed fortified human milk and whey-dominant formula, respectively, suggest that the synthesis of specific functional proteins should be examined more closely than has been done in previous studies seeking to establish optimal intakes.

Acknowledgments. The authors thank M. P. Thotathuchery for technical assistance, P. Burns for nursing assistance, J. Hopkinson for lactation management, J. K. Fraley and E. O. Smith for data and statistical advice, E. R. Klein for editorial assistance, and N. Haley and M. E. Lewis for manuscript preparation.

\section{REFERENCES}

1. Committee on Nutrition, American Academy of Pediatrics 1985 Nutritional needs of low-birth-weight infants. Pediatrics 75:976-986

2. Berger HM, Scott PH, Kenward C, Scott P, Wharton BA 1979 Curds and whey proteins in the nutrition of low birthweight babies. Arch Dis Child 54:98104

3. Bezkorovainy A 1977 Human milk and colostrum proteins: a review. J Dairy Sci 60:1023-1037

4. Jenness R 1974 The composition of milk. In: Larson BL, Smith VR (eds) Lactation-A Comprehensive Treatise. Academic Press, New York, pp 3107

5. Rassin DK, Gaull GE, Heinonen K, Raiha NCR 1977 Milk protein quantity and quality in low-birthweight infants: II. Effects on selected aliphatic amino acids in plasma and urine. Pediatrics 59:407-422

6. Scott PH, Berger HM, Wharton BA 1985 Growth velocity and plasma amino acids in the newborn. Pediatr Res 19:446-450

7. Anderson GH, Bryan H, Jeejeebhoy KN, Corey P 1977 Dose response relationships between amino acid intake and blood levels in newborn infants. Am J Clin Nutr 30:1110-1121

8. Raiha NCR, Heinonen K, Rassin DK, Gaull GE 1976 Milk protein quantity and quality in low-birthweight infants: I. Metabolic responses and effects on growth. Pediatrics 57:659-674

9. Natelson S, Penniall R, Crawford WL, Munsey FA 1955 Noncasein protein to casein ratio of feeding formulas-effect on blood component levels in norma infants. Am J Dis Child 89:656-668 
10. Gaull GE, Rassin DK, Raiha NCR, Heinonen K 1977 Milk protein quantity and quality in low-birth-weight infants: III. Effects on sulfur amino acids in plasma and urine. J Pediatr 90:348-355

11. Rassin DK, Gaull GE, Raiha NCR, Heinonen K 1977 Milk protein quantity and quality in low-birth-weight infants: IV. Effects on tyrosine and phenylalanine in plasma and urine. J Pediatr 90:356-360

12. Schanler RJ, Garza C, Nichols BL 1985 Fortified mothers' milk for very low birthweight infants: results of growth and nutrient balance studies. J Pediatr 107:437-445

13. Malloy MH, Rassin DK, Gaull G 1981 A method for measurement of free and bound plasma cyst(e)ine. Anal Biochem 113:407-415

14. Mondino A, Bongiovanni G, Fumero S 1975 A new approach for obtaining total tryptophan recovery in plasma samples deproteinized with sulfosalicylic acid. J Chromatogr 104:297-302

15. Svanberg U, Aebsi-Medlin M, Ljunggvist B, Olsson M 1977 Breast milk composition in Ethiopian and Swedish mothers. III. Amino acids and other nitrogenous substances. Am J Clin Nutr 30:449-507
16. Ronnholm KAR, Simell O, Siimes MA 1984 Human milk protein and medium-chain triglyceride oil supplementation of human milk: plasma amino acids in very low-birth-weight infants. Pediatrics 74:792-799

17. Pohlandt F 1978 Plasma amino acid concentrations in umbilical cord vein and artery of newborn infants after elective cesarean section of spontaneous delivery. J Pediatr 92:617-623

18. McIntosh N, Rodeck $\mathrm{CH}$, Heath $\mathrm{R} 1984$ Plasma amino acids of the midtrimester human fetus. Biol Neonate 45:218-224

19. Pardou A, Vuye A, Vis HL 1982 Plasma amino acid patterns, one and two hours after continuous naso-gastric alimentation of low birth weight infants fed two types of milk during the first month of life. Biol Neonate 41:57-62

20. Ronnholm KAR, Siimes MA 1985 Haemoglobin concentration depends on protein intake in small preterm infants fed human milk. Arch Dis Child 60:99-104

21. Young VR, Scrimshaw NS 1977 Human protein and amino acid metabolism and requirements in relation to protein quality. In: Bodwell CE (ed) Evaluation of Proteins for Humans. AVI Publishing Co, Westport, CT, pp 11-59 